\title{
Las refugiadas políticas colombianas en España: aportes para la disciplina de Trabajo Social de los enfoque de género e intercultural
}

\author{
Elena Mut Montalva ${ }^{1}$
}

Recibido: 30/04/2017 / Revisado: 18/06/2017 / Aceptado: 02/11/2017

Resumen. El propósito del presente artículo es aportar conocimientos para la disciplina de Trabajo Social, específicamente en su praxis con mujeres y en la intervención colectiva con el enfoque de género y el enfoque intercultural. Partiendo de la idea de que todo conocimiento es situado, la presente investigación feminista subraya la importancia de pensar desde la perspectiva de las vidas de los grupos excluidos, en este caso de las refugiadas colombianas exiliadas en España, para visibilizar sus aportaciones a la defensa de los derechos humanos, al desarrollo humano sostenible y a la superación de la vulnerabilidad, a través de procesos de empoderamiento y de constitución de redes de apoyo. Para ello se reflexiona, por una parte, sobre la complejidad del refugio político y, por la otra, sobre sus prácticas asociativas. Aunque el drama del exilio es un elemento que atraviesa sus trayectorias vitales relegándolas a una posición subalterna, se analizan sus estrategias de resistencia, resiliencia y empoderamiento a través de su activismo en asociaciones y plataformas. De esta forma, se profundiza en el significado de la diversidad como característica inherente a la sociedad actual en las que convivimos con personas de diversas culturas, valores, creencias religiosas, identidades étnicas y de género, orientaciones sexuales, prácticas sociales, ideologías políticas y estilos de vida y, en particular, en el significado de las contribuciones de las refugiadas colombianas asentadas en España.

Palabras clave: Trabajo Social; refugiadas políticas; competencia intercultural; género; derechos humanos.

\section{[en] Colombian political refugees in Spain: contributions to the field of Social Work from gender and intercultural perspectives}

\begin{abstract}
The purpose of this article is to provide knowledge in the field of social work, specifically in terms of its practice with women and group intervention with a gender and intercultural focus. Based on the idea that all knowledge is situated, this feminist research underlines the importance of thinking from the perspective of the lives of excluded groups, in this case of Colombian refugees exiled in Spain, in order to make visible their contributions to the defence of human rights, to sustainable human development, and to overcoming vulnerability, through processes of empowerment and creation of support networks. There is reflection on the complexity of political refuge and on its associative practices. Though the drama of exile is an element that affects their life pathways and places them in a subordinate position, the article analyses refugees' strategies for resistance, resilience and empowerment through activism via associations and platforms. It thereby examines the meaning of diversity as a characteristic that is inherent to contemporary society, in which we coexist with people of diverse cultures, values, religious beliefs, ethnic and gender identities, sexual orientations, social practices, political ideologies and lifestyles, and in particular the meaning of contributions from Colombian refugees settled in Spain.
\end{abstract}

Key words: social work; political refugees; intercultural competition; gender; human rights.

Sumario: 1. Introducción sobre las líneas que han orientado la investigación, el contexto y los objetivos. 2. Metodología y aproximación teórica y conceptual. 3. Resultados del estudio relativos a las características de las trayectorias vitales de las refugiadas. 4. Las principales conclusiones de la investigación. 5. Referencias bibliográficas.

Cómo citar: Mut Montalva, E. (2018) Las refugiadas políticas colombianas en España: aportes para la disciplina de Trabajo Social de los enfoque de género e intercultural, en Cuad. trab. soc. 31(1), 59-68. 


\section{Introducción sobre las líneas que han orientado la investigación, el contexto y los objetivos $^{2}$}

Entre las paradojas y contradicciones que encierra la globalización encontramos que, al tiempo que se incrementan los desplazamientos voluntarios a escala planetaria de una ciudadanía cosmopolita, los conflictos armados provocan desplazamientos forzosos de importantes poblaciones. Dichas contradicciones pueden impulsar cambios sociales y políticos en la medida en que las actoras y actores sociales influyan en la acción política a escala internacional. Este es el caso de determinados colectivos que exigen tener un papel político y el reconocimiento de sus derechos, como ocurre con las refugiadas colombianas en España.

Las migraciones inciden de forma protagónica en los procesos de transformación social, política, económica y cultural de las sociedades y configuran conexiones y redes entre lugares distantes y culturas diferentes que deben considerarse en el diseño de las políticas sociales y en los programas de intervención e inclusión social ${ }^{3}$.

Las elevadas cifras de personas exiliadas y desplazadas de Colombia evidencian que asistimos a una de las mayores catástrofes colectivas de América, con siete millones de personas afectadas. El exilio constituye una de las mayores tragedias humanas que el conflicto armado ha provocado. Asimismo, dicho conflicto ha impactado gravemente en las vidas de las mujeres, empeorando sus condiciones de vida y con el agravante de la violencia. Como las guerras no son neutrales con el sexo, muchas mujeres han sufrido diversos tipos de violencia (entre ellas la violencia sexual), persecuciones y amenazas y además para algunos grupos armados, las organizadas en movimientos de mujeres ${ }^{4}$ han sido el objetivo de una estrategia de agresiones.
El VI Informe de la Mesa "Mujer y conflicto armado" describe que las violaciones contra las defensoras de derechos constituyen uno de los hechos de mayor gravedad, pues revestía connotaciones individuales y colectivas que las lesionaban de manera irreparable. Posteriormente, el Estado colombiano reconoció la necesidad de adoptar un "enfoque diferencial, de género, de derechos y equidad para las mujeres", 5 por lo que en el 2012, a través de la Unidad para la Atención y la Reparación Integral de las Víctimas (UARIV), ofreció a dos organizaciones de mujeres -la Organización Femenina Popular (OFP) y la Asociación de Mujeres Campesinas, Negras e Indígenas de Colombia (ANMUCIC) - cuyas militantes fueron sistemáticamente perseguidas y asesinadas, la reparación colectiva como un gesto de reconocimiento de la responsabilidad del Estado en los crímenes, por acción u omisión. Pese a este avance, en la actualidad todavía no es posible el retorno seguro a su país, pues los asesinatos y las agresiones contra defensores de derechos se han incrementado en el año 2017.

El objeto de la investigación ha sido el estudio de las prácticas asociativas para la defensa de los derechos humanos que las refugiadas colombianas en España despliegan en el espacio local, nacional y trasnacional, y sus implicaciones para la disciplina del Trabajo Social. Dicho estudio ha estado orientado por los siguientes objetivos generales:

1. Caracterizar el activismo de las refugiadas colombianas en España en la promoción y defensa de los derechos humanos, descubriendo sus énfasis y contenidos.

2. Analizar las prácticas asociativas en su exilio y sus aportaciones para la construcción de la paz, al desarrollo humano y la igualdad.

Esta investigación toma como punto de partida la tesis doctoral de la autora (consultar bibliografía), a la que se han aportado nuevas reflexiones y resultados aplicados a la praxis y la disciplina del Trabajo Social.

Por ejemplo, como la realidad de migrantes y refugiadas/os es compleja y está vinculada a más de un Estado-nación, en el diseño de las políticas sociales y los planes de servicios sociales no se considera que muchas de estas personas están sosteniendo dos hogares: uno en España y el otro en Colombia, por lo que en sus protocolos no se contemplan estos elementos de mayor vulnerabilidad.

4 Las integrantes de la Asociación de Mujeres Campesinas, Negras e Indígenas de Colombia (ANMUCIC) y la Organización Femenina Popular (OFP) sufrieron sistemáticamente amenazas, y sufrieron secuestros, violaciones y asesinatos. Contra las mujeres de la OFP se cometieron más de ciento cuarenta crímenes y de ANMUCIC fueron asesinadas más de 40 mujeres. Varias de las refugiadas entrevistadas fueron dirigentes de estas organizaciones.

El enfoque diferencial implica diseñar y hacer transversales acciones de prevención, atención, asistencia y reparación integral en el marco del conflicto interno armado, de acuerdo con la gravedad de las consecuencias del conflicto para las mujeres y teniendo en cuenta las solicitudes realizadas por las mujeres y por las organizaciones de víctimas. 
3. Reflexionar sobre la pertinencia del enfoque intercultural ${ }^{6} \mathrm{y}$ de género ${ }^{7}$ para la disciplina del Trabajo Social, con el estudio del caso de las refugiadas colombianas.

\section{Metodología y aproximación teórica y conceptual}

La metodología utilizada es de carácter cualitativo y las tres técnicas investigación desplegadas han sido: el análisis documental y bibliográfico; las entrevistas han constituido el instrumento principal para la recolección de la información, realizándose un total de $39^{8} \mathrm{y}$, por último, la observación participante realizada en diversos espacios asociativos en las que participan las refugiadas colombianas.

Las características sociodemográficas de las refugiadas entrevistadas son: sus edades oscilan entre los 25 y los 67 años; la mayoría son madres, de clase media baja y de origen rural de los departamentos: Valle del Cauca, Norte de Santander, Santander, Cundinamarca y Caldas. Las Comunidades Autónomas en la que residen en su exilio son: Comunidad Valenciana, Castilla la Mancha, Madrid, Cataluña y País Vasco. La mayoría han cursado estudios superiores y su nivel académico y situación laboral en Colombia contrasta con su situación laboral en el exilio, pues todas ellas han encontrado muchas dificultades para obtener un empleo: en su mayoría son trabajadoras temporales a tiempo parcial, generalmente en el empleo doméstico y en el sector servicios, con largos periodos de desempleo.

El feminismo es la perspectiva teórica que cruza transversalmente la investigación que se enriquece con el uso de otros conceptos teóricos clave que orientan el estudio: la migración y el refugio; el transnacionalismo, el enfoque intercultural y de género y, por último, el activismo en la promoción y defensa de los derechos humanos.
La gravedad de la actual crisis del refugio que atraviesa Europa hace necesario que se incorporen los conocimientos y resultados de esta investigación para su aplicación en el ámbito del Trabajo Social. Vivimos en una sociedad de inmigración y refugio, por lo que nuestras ciudades son ahora territorios de diversidad profunda donde la multiculturalidad es "un rasgo estructural significativo, no solo desde el punto de vista cuantitativo, sino también cualitativamente" (de Lucas, 2012, p.16). En este sentido, la integración en la diversidad constituye un reto insoslayable, pues convivir juntos, siendo diferentes, complejiza el abordaje en el diseño y la gestión de las políticas públicas $\mathrm{y}$, particularmente, en los servicios sociales. La diversidad en su dimensión cultural y de género, a la vez que supone una fuente de enriquecimiento para las sociedades de acogida, genera también importantes tensiones identitarias y culturales que repercuten en la cohesión social.

\section{Resultados del estudio relativos a las características de las trayectorias vitales de las refugiadas}

García Canclini (1999) señala la importancia de tener en cuenta a las personas que reproducen y padecen la globalización neoliberal, pues incluirlas en los análisis permite tener en cuenta tres importantes aspectos, estrechamente conectados entre sí y con la globalización neoliberal, como son: el drama, la responsabilidad y la posibilidad de reorientar el itinerario. En el caso de las refugiadas colombianas: el drama se refiere a la tragedia por su condición de exiliadas; la responsabilidad se relaciona con las empresas multinacionales, las élites locales y al gobierno colombiano por la impunidad y la falta de garantías en la protección a las personas y, en último lugar, la posibilidad de reorientar el itinerario está referido a la capacidad de agencia, al grado de empoderamiento y a la

\footnotetext{
En referencia a este enfoque, lo que hace a una persona culturalmente competente es la capacidad de entender con exactitud y de responder eficazmente a personas de diferentes culturas. Y esta competencia cultural implica una combinación de conocimientos, habilidades y actitudes.

La irrupción de la perspectiva de género en las ciencias sociales ha provocado cambios que ya parecen irreversibles e introduce la posibilidad de examinar, desde otra óptica, la realidad que nos rodea y percibir así la existencia de los obstáculos que encuentra el desarrollo de la igualdad en los distintos ámbitos sociales, culturales, políticos, económicos, etc. Es aquella que tiene en cuenta las diferencias y desigualdades entre hombres y mujeres, por lo tanto, visibiliza el modo en que el género puede afectar a la vida y a las oportunidades de las personas para resolver sus problemas y dificultades.

824 biográficas a refugiadas (a través de las que se han elaborado 7 historias de vida); y 15 en profundidad (6 a informantes clave expertas, españolas y colombianas de las plataformas en las que participan las refugiadas y 9 a trabajadoras sociales de los Centros Municipales de Servicios Sociales y Centros de Salud en Valencia).
} 
capacidad de resiliencia de las exiliadas. Estos tres elementos se hacen visibles en las trayectorias vitales de las refugiadas y la posibilidad de reorientar el itinerario constituye el grueso de la investigación, pues las refugiadas se constituyen como agentes sociales que influyen en las políticas públicas colombianas y en las de la sociedad de acogida. Como ejemplo tenemos el siguiente testimonio:

Los primeros años en el país de acogida tuvimos que minimizar nuestro accionar, y limitarnos a las actividades que ( ...) podíamos desarrollar en España. Con el tiempo, se ha ido recuperando el sentimiento de empoderamiento e incidencia sociopolítica en el país de origen y en el que nos ha acogido (Alba Teresa).

El primer rasgo que las define está referido a la transformación de su identidad. El exilio produce una crisis en su identidad, debido a la dureza de la experiencia vivida a través de un corolario de bruscas rupturas respecto al país de origen, a la que se suma el complejo proceso de adaptación en la sociedad de acogida (Castaño y Rueda, 2010). Experimentan: choque cultural, baja autoestima, sensación de estar con las manos vacías y el fuerte deseo del regreso a su país. Sentimientos que se entremezclan con una progresiva transformación identitaria para, finalmente, reconstruir una nueva identidad compleja e híbrida compuesta por el cruce de culturas y de sentidos de pertenencia. También han adquirido una identidad colectiva nueva y específica: la de ser mujeres refugiadas, que ha cristalizado en la conformación de la Colectiva de Mujeres Refugiadas, Exiliadas y Migradas en España ${ }^{9}$, que supone un espacio en el que se sienten identificadas tanto por su experiencia en Colombia, como en el exilio.

El segundo rasgo característico es su intensa participación política, asociativa y supraasociativa. A lo largo de su exilio se han involucrado en un intenso activismo a nivel local, autonómico, nacional e internacional, que ha supuesto una estrategia para la denuncia y la visibilización de las violaciones a los derechos humanos. También han identificado el escenario de finalización de la guerra como una oportunidad para reclamar sus derechos como víctimas y como exiliadas, así como su reconocimiento como interlocutoras (capacidad de agencia) ante el gobierno colombiano. Dos de las refugiadas lo describen así:

El exilio también puede ser una oportunidad para visibilizar internacionalmente el conflicto y sus consecuencias. Podemos acceder a la justicia internacional, documentar casos de violación de derechos humanos y realizar denuncias, (...) incidencia política (Leonora).

Hemos insistido para que en el proceso de paz se incluyan las voces de las mujeres refugiadas políticas y exiliadas y de las víctimas, exigiendo el cumplimiento de la Resolución 1325 de Naciones Unidas para garantizar la participación de las mujeres en los acuerdos de paz como sujetas políticas que somos y vinculando la participación de Mujeres víctimas y protagonistas en el trabajo por la Paz de Colombia para alcanzar justicia para las mujeres y el reconocimiento de todos sus derechos incorporándolos a los acuerdos emanados de la agenda temática (Alba Teresa).

En tercer rasgo lo constituye el feminismo práctico, pues han visibilizado la grave situación de vulneración de derechos de las mujeres en Colombia y las dificultades de las exiliadas. Con sus actividades de sensibilización social e incidencia política han incluido la voz de las personas en el exilio para que sus propuestas sean tenidas en cuenta en el proceso de construcción de la paz en el país. Pese a que sus actuaciones para la ampliación de la democracia y la igualdad entre mujeres y hombres la hemos observado tanto en su país de origen (en los procesos de desarrollo en las comunidades campesinas e indígenas; en el acceso a la propiedad de la tierra de las mujeres; en la defensa de los derechos de las mujeres; etc.), como en su exilio, la mayoría no se definen como feministas, aunque muestran una gran sensibilidad hacia la problemática de las mujeres que sale a la luz en su activismo atravesado por un feminismo práctico.

Por último, en cuanto a la diferenciación interna entre las refugiadas de la Colectiva destacamos: en primer lugar, que en su gran mayoría son de izquierdas, con diferentes militancias

La Colectiva se constituyó en el año 2005, y participan refugiadas colombianas que residen en Madrid, Barcelona, Valencia, Alicante, Villena, Albacete, Bilbao, y otras ciudades españolas. Es la asociación de refugiadas colombianas más importante y mejor organizada de Europa. 
políticas tanto en Colombia (en el Partido Verde, Unión Patriótica, Partido comunista, Polo Democrático), como en España (en Podemos, Izquierda Unida y PSOE). Es por ello por lo que sus diferencias no las encontramos en su ideología o en la estrategia compartida de adoptar el enfoque de derechos humanos, sino en el método de trabajo y en la selección de las prioridades temáticas (han existido conflictos por sus posiciones diferentes respecto a que la estrategia de género sea la prioritaria). Otra diferencia la encontramos en sus distintas posiciones respecto a la necesidad de ampliar el número de integrantes y de fortalecer su organización a nivel internacional (Ecuador), en la que no todas están de acuerdo y señalan, unas: "que todavía la Colectiva no está suficientemente consolidada" y otras: "que nunca nos vamos a sentir totalmente consolidadas y por ello es necesario ampliar" (Leonora).

\section{Las principales conclusiones de la investigación}

Se presentan agrupadas en dos grandes bloques: el primero analiza los retos del futuro para la disciplina y práctica del Trabajo Social, debido al incremento de la diversidad como característica inherente a todas las sociedades, pues convivimos con personas que atesoran diversas culturas, valores, creencias religiosas, identidades étnicas y de género, orientaciones sexuales, ideologías políticas, condiciones sociales y estilos de vida. Y, en el segundo, se presentan las aportaciones sobre la complejidad derivada de la condición del refugio y del activismo de las refugiadas.

La primera conclusión es que, en la actualidad, la disciplina del Trabajo Social afronta nuevos desafíos, derivados del intenso proceso de globalización que la atraviesa y que condiciona su intervención en contextos mucho más diversos que cuando surgió dicha disciplina. Este hecho nos lleva a cuestionar los paradigmas del Trabajo Social y su eficacia en contextos culturalmente diversos. Por ello, los resul- tados presentados muestran tendencias sobre las características del refugio y el activismo de las colombianas asentadas en España que son de utilidad para su aplicación en el ejercicio profesional, debido a sus singulares características e implicaciones para la mejora de dicha práctica profesional.

Paralelamente al incremento de les migraciones, las mujeres también se han convertido en agentes activos y visibles de estos procesos migratorios. Después de tres décadas de un significativo proceso acogida de inmigrantes en España, es importante reflexionar tanto en los procesos de inclusión y exclusión de esta población, como de construcción de estas nuevas identidades híbridas. El nuevo perfil de usuarias y usuarios de servicios sociales se caracteriza por la diversidad étnica y de nacionalidad de origen ${ }^{10}$, así como por la feminización de las desigualdades, que se explican por los mayores índices de pobreza y exclusión en los tres principales ejes de integración: el mercado laboral, el Estado de bienestar y las redes personales. Ello tiene su correlato en la sobrerrepresentación de las mujeres en la franja de pobreza, debido a diversos factores condicionados por la nacionalidad y el género, entre ellos: hogares sin ingresos encabezados por mujeres ${ }^{11}$ y con adolescentes a cargo; las altas tasas de maternidad precoz; la prolongada exclusión de las mujeres de los circuitos formales del mercado de trabajo; su inserción laboral intermitente, que tiene como consecuencia la escasa cotización a la seguridad social para acceder a las prestaciones contributivas, lo que las limita a prestaciones asistenciales e incrementa su vulnerabilidad frente a la violencia de género así como su interrelación con los procesos de exclusión ${ }^{12}$ (irregularidad de ingresos, redes sociales poco densas, problemas de salud, vivienda precaria); etc. (Agrela, Gutiérrez y Fernández, 2017, p. 58). En este sentido, una de las refugiadas señala que:

A las mujeres exiliadas se nos condena a la muerte social. Muchas de nosotras hemos pasado de ser sujetos de protección de los derechos humanos a objetos de protección. Dejando atrás

10 La nacionalidad de origen está imbricada en el nuevo contexto de la sociedad de acogida, pues la adquisición de nuevas identidades híbridas por las personas migrantes y refugiadas vuelven más compleja la dualidad de "ser de aquí o de allá".

11 Las trabajadoras sociales entrevistadas señalan que las mujeres inmigrantes con hijos a cargo son el colectivo que más necesidades presenta.

12 Por ejemplo, es necesario reconocer que los indicadores de riesgo para las víctimas de violencia de género en el acceso a los sistemas de protección no son los mismos según la nacionalidad o la etnia, ya que este disminuye si el agresor está en la cárcel, situación que no es homogénea en todas las nacionalidades o etnias. Ello conlleva a un déficit en las intervenciones y en los protocolos protección a víctimas, ya que no incluyen la diversidad social y cultural. 
Colombia, también dejamos atrás todo un proyecto de vida. Lejos de nuestro país, sufrimos valores culturales diferentes, pérdidas afectivas, dificultades para un reconocimiento laboral o social como el que teníamos en Colombia, nuevos procesos de aprendizaje y relacionales... creo que hay una falta de atención integral a la población refugiada (Leonora).

Este incremento de la diversidad respecto a las características sociales y culturales de las y los usuarios de los servicios públicos debe acompañarse de mejores competencias interculturales de sus profesionales, así como de formación adecuada en sus distintas dimensiones para garantizar una atención pertinente y de calidad en los distintos niveles de la intervención social. Para abordar los retos de la diversidad, Cohen-Emerique plantea el término enfoque intercultural y no competencia, porque entiende que este incluye un proceso dinámico y complejo que incorpora las competencias en las diferentes etapas que define como: descentramiento, descubrimiento del marco de referencia del otro; negociación y mediación. La capacidad de desarrollar dichas etapas, es lo que la autora define como enfoque intercultural y permite el acceso a conocimientos teóricos sobre la multiplicidad y la complejidad de los factores que interfieren en cualquier encuentro intercultural y, por el otro, delimitar las zonas sensibles que aparecen en las interacciones de ayuda y acompañamiento a las personas migrantes y refugiadas:

Estas zonas se denominan sensibles porque, por un lado, para el trabajador social son una fuente de incomprensiones asociadas a juicios de valores erróneos; y por otro, para el usuario, representan un no-reconocimiento de sus identidades, que son múltiples y únicas al mismo tiempo (2013, pp. 11-38).

Este enfoque saca a la luz la rigidez de determinados modelos y principios profesionales que no se adaptan a las estructuras familiares no occidentales y migrantes. Asimismo, nos revela la necesidad de replantearse las modalidades de intervención con los migrantes: formación, reflexiones, intercambios de experiencias entre personas del mismo equipo. Dicha autora señala también que todos los discursos sobre competencias interculturales empiezan con la necesaria empatía por la alteridad y al enriquecimiento que se obtiene de la diversidad, aunque lo realmente importante es indicar que las relaciones y la comunicación intercultural pueden ser difíciles, porque implican procesos muy complejos. También pueden ser una fuente de malos entendidos debido a los etnocentrismos, androcentrismos y los prejuicios inherentes a los diferentes orígenes culturales, y pueden chocar con los principios de tolerancia hasta el punto de ser negadas o escondidas tras una perspectiva de carácter universalista (idem).

Con los hallazgos obtenidos se ha visibilizado el refugio político del colectivo colombiano y, específicamente, el de las mujeres, configurando así una imagen más completa de la compleja situación de las personas refugiadas en España. Además, dada magnitud de la actual crisis del refugio en Europa, estos conocimientos resultan hoy en día necesarios para las y los profesionales de Trabajo Social. Entre los retos identificados para su aplicación la práctica profesional destacamos:

1. La necesidad de una formación en la migración y el refugio como fenómeno global atravesado por una perspectiva bidireccional en la que existe una interacción continúa entre la sociedad de origen y la de acogida, que tiene repercusiones en la valoración de los casos y en la elaboración de protocolos de actuación;

2. La escasa presencia en los protocolos de intervención del enfoque de la diversidad cultural y, la todavía más escasa existencia de protocolos que aúnen los dos enfoques: de género y de diversidad cultural;

3. La necesidad de reflexionar y atender las demandas sobre las transformaciones identitarias de las personas migrantes $\mathrm{y}$, consecuentemente, en la conformación de identidades híbridas, que conllevan importantes crisis;

4. Considerar el potencial y la significativa contribución a la superación de la vulnerabilidad social a través de los procesos de empoderamiento y de constitución de redes de apoyo y de ayuda mutua que caracterizan a la mayoría de las refugiadas;

5. Prestar la adecuada atención a las necesidades de atención psicosocial y psiquiátrica a mujeres que han sufrido graves secuelas por las situaciones vividas por la vulner- 
ación de derechos en su país de origen (amenazas, secuestros, acoso, agresiones sexuales, violaciones en grupo, etc.), así como en el proceso de tránsito en la migración y en dotar de las herramientas para la educación de hijas/os reagrupados que han vivido durante años separados de sus madres/padres.

Las mujeres afrontan diversas discriminaciones como: mujeres, inmigrantes, refugiadas, por situación administrativa irregular; por su etnia, religión y, además, están condicionadas por estereotipos propios de la cultura de origen y los de la cultura receptora, por ello comparten una serie de características generales que hay que tener en cuenta a la hora de la intervención:

1. El concepto de familia extensa y construcción de redes sociales, desde donde pivotan el resto de valores.

2. La prevalencia y jerarquía de los hombres en la organización de la vida social y en la familia.

3. La solidaridad en situaciones extremas como dificultades económicas, enfermedad o fallecimiento de algún miembro.

4. Preeminencia de valores extremadamente patriarcales.

Por ello, desde el enfoque de género en la intervención social se deben asumir los siguientes objetivos: 1) analizar, reflexionar y reducir los efectos del sexismo en las usuarias de los servicios sociales a través de la potenciación de la autonomía; y 2) eliminar la discriminación subyacente en la práctica profesional incorporando los puntos de vista de las mujeres; investigar los aspectos de género en la vida social cotidiana; considerar diferenciadamente sus necesidades y capacidades y los impactos de la discriminación en el trabajo, pobreza, cambios familiares, etc.; distinguir las vivencias comunes y diferentes entre las mujeres; obtener un conocimiento más científico de la realidad; prestar atención a las políticas discriminatorias y no continuar con el mantenimiento de roles, así como la eliminación de las actitudes discriminatorias interiorizadas.
También es de utilidad la estrategia de la interseccionalidad para hacer frente a las desigualdades entre mujeres y hombres, por su carácter proactivo y porque trata las desigualdades estructurales enfrente a lo individual.

El análisis interseccional tiene como objetivo revelar las variadas identidades, exponer los diferentes tipos de discriminación y desventaja que se dan como consecuencia de la combinación de identidades (experiencias sustantivamente diferentes en función de múltiples pertenencias: clase, género, etnia, discapacidad) (AWID, 2004).

El empoderamiento, como noción tomada de los movimientos sociales es relevante para la trasformación de las prácticas profesionales en el Trabajo Social.

En la medida en que toma en cuenta las condiciones sociales y se basa en las dinámicas individuales y colectivas, en una cadena de equivalencias que articula toma de conciencia o conciencia crítica, capacitación, autoestima, autodeterminación, reciprocidad entre cliente y trabajador social, trabajo colectivo (Bacqué y Biewener, 2016, p. 42).

Asimismo, advierten estas autoras del peligro de que se interprete únicamente como una propuesta centrada en la responsabilidad de los individuos.

La combinación de ambos enfoques, intercultural y de género, permite:

1. la identificación de las diferencias en los roles e identidades de género y su desigual valoración económica y social en los diferentes entornos socioculturales;

2. la identificación de las dificultades que experimentan las mujeres para acceder y controlar los recursos necesarios para satisfacer sus necesidades en los diferentes países, el de origen y el de acogida ${ }^{13} y$,

3. la identificación de sus dificultades para tomar las decisiones necesarias para desarrollar libremente sus proyectos vitales.

Tras la constatación de la necesidad de incrementar la formación para las trabajadoras y

Las/os profesionales de Trabajo Social entrevistados han destacado las limitaciones de los servicios sociales para entender las necesidades de las madres inmigrantes con familia en su país de origen y en el de acogida. 
trabajadores sociales en materia de diversidad social y cultural, se concluye la importancia de adquirir nuevas competencias y una actualización permanente desde el enfoque intercultural y de género que les doten de los conocimientos, habilidades y actitudes que incrementen su capacidad de entender con exactitud y de responder eficazmente a las necesidades de personas provenientes de diferentes culturas, así como de comprender el significado diferencial del género y de sus riesgos particulares en diferentes culturas y etnias.

En el segundo bloque de conclusiones, que se divide a su vez en cuatro apartados, abordamos las características del activismo de las refugiadas para generar una reflexión crítica sobre cuál es el modelo de convivencia y de sociedad a la que aspiramos y sobre la mejora de la formación de las y los profesionales en el ámbito de los servicios sociales en la atención a la diversidad para que nuestras ciudades y servicios públicos sean espacios integradores, de encuentros y reconocimientos en un sentido compartido de comunidad y de sociedad inclusiva. A continuación desarrollamos estos cuatro apartados que ahondan en la complejidad del refugio:

1. Las refugiadas han puesto de relieve las múltiples violaciones de derechos que han sufrido en el contexto de la guerra y que ha originado su exilio. Estas se han organizado en asociaciones, plataformas y partidos políticos a través de los que han mostrado su posicionamiento en contra de la guerra y han reivindicado que su voz esté presente en la construcción de la paz. Para ello, a través de la Colectiva, están desplegando una serie de iniciativas en las que exponen sus propuestas específicas como mujeres víctimas y exiliadas, que han remitido a la ONU Mujeres, al gobierno colombiano y a la Mesa de negociaciones de paz para la construcción de una paz sostenible y duradera en el país.

2. Otro rasgo definitorio es su activismo múltiple y el elevado grado de participación en diferentes plataformas ${ }^{14}$ en España. Su activismo adquiere una fuerte dimensión política ya que convierten los espacios asociativos en espacios de reivindicación política a través de campañas de denuncia sobre la situación de vulneración de derechos. Esta intensa participación en asociaciones $\mathrm{y}$ entidades supraasociativas ${ }^{15}$ caracteriza su activismo en el exilio y nos lleva a afirmar que el activismo ocupa un lugar central en sus vidas. Su exilio les ha permitido desenvolverse en un contexto de lucha por los derechos de las refugiadas en el espacio global, por lo que su experiencia como refugiadas, a pesar de su dureza, ha supuesto para ellas una fuente de conocimiento y de aprendizaje al actuar como agentes activos en la transformación de la realidad social y política.

3. Las exiliadas se configuran como impulsoras en la transformación de las relaciones desiguales de poder por su implicación en la construcción de una sociedad igualitaria. Presentan un elevado grado de implicación como dirigentes de importantes asociaciones y plataformas en defensa de los derechos de las mujeres y se ha constatado su articulación como sujeto político: las mujeres exiliadas a través de la Colectiva. Este intenso activismo lo ubicamos en el feminismo práctico, pues la creación de la Colectiva en España responde a la necesidad sentida por estas mujeres para reconstruir y fortalecer su identidad, para exigir medidas para satisfacer sus necesidades en el exilio y, finalmente, para solicitar las garantías y derechos para su regreso seguro a Colombia.

4. Su activismo se caracteriza por su transnacionalidad, por sus vínculos constantes en la defensa de los derechos de las mujeres colombianas y de las exiliadas ${ }^{16}$ que fomentan una óptica relacional entre las sociedades de origen y de acogida y favorecen el trabajo conjunto entre nuevos y viejos agentes sociales en pro de la defensa

14 Algunas de ellas han adquirido un relevante protagonismo en el movimiento asociativo, un ejemplo es una activista que ha sido coordinadora de la plataforma de ONGD en Albacete, obteniendo premios y reconocimientos.

15 Entre ellas, la Coordinación Valenciana de Solidaridad con Colombia y, la más significativa, la Mesa de Apoyo a la defensa de los Derechos Humanos de las Mujeres y la Paz en Colombia compuesta por 24 organizaciones y plataformas españolas y colombianas. 
de los derechos humanos. Los marcos relevantes de estructuración social de las refugiadas no son solo los espacios locales, pues también salen a la luz sus prácticas en los contextos autonómico, nacional e internacional. La frecuencia y la intensidad de sus interacciones nos indican que están sentando las bases de una nueva ciudadanía transnacional. Y ello se hace patente en las actividades que despliegan hacia la opinión pública y que contribuyen a que las personas autóctonas se planteen nuevas preguntas y redescubran y comprendan las características de la compleja sociedad global.

Lo más singular es su significativo protagonismo en las distintas plataformas transnacionales en la defensa de los derechos de las mujeres colombianas, las cuáles resultan funcionales para el logro de sus objetivos políti- cos, pues son espacios flexibles que permiten el tránsito hacia otro tipo de experiencias participativas, así como de crear alianzas con la sociedad civil y las instituciones locales, regionales, nacionales e internacionales.

Concluimos que el activismo de las refugiadas se encuentra permeado por un ideario en el que juegan un papel destacado la moral y los valores, así como su interés en la esfera de lo social desde un posicionamiento ético que las sitúa al lado de los colectivos más vulnerables y excluidos. La praxis que despliegan se sustenta y cobra sentido a partir de dicho posicionamiento ético. Esta visión moral de la realidad justifica la trascendencia de su activismo en la ampliación las libertades en los diferentes territorios por los que transitan para la consecución de un mundo fundamentado en los valores de justicia social, paz e igualdad como parte de un proyecto vital que dota de sentido a sus vidas.

\section{Referencias bibliográfica}

Agrela, Belén, Gutiérrez, Camino y Fernández, Teresa (2017). Repensar la ética en Trabajo Social desde una perspectiva de género. Cuadernos de Trabajo Social, 30(1), 51-64.

Asociación para los Derechos de la Mujer y el Desarrollo. (AWID) (2004). Interseccionalidad: una herramienta para la justicia de género y la justicia económica. Derechos de las mujeres y cambio económico, 9.

Bacqué, Marie-Hélène y Biewener, Carole (2016). El empoderamiento. Una acción progresiva que ha revolucionado la política y la sociedad. Barcelona: Gedisa.

Castaño, Leonora y Rueda, Pilar (2010). Vivir con dignidad. Historia de vida de Leonora Castaño Cano. Bogotá: Corcas Editores.

Cohen-Emerique, Margalit (2013). Por un enfoque intercultural en la intervención social. Educación Social. Revista de Intervención Socioeducativa, 54, 11-38

Cucó Giner, Josepa (2011). Feminismo y militancia política. El feminismo del MC y de las organizaciones postemeceras. Ponencia en el XII Congreso de la FAAEE: Etnografiando resistencias. León del 5-9 de septiembre.

De Lucas, Javier (2012). Sobre los fundamentos de la igualdad y del reconocimiento. Un análisis crítico de las condiciones de las políticas europeas de integración ante la inmigración. En: J. de Lucas et al., Inmigración e integración en la UE. Dos retos para el s. XXI (pp.11-91). Vitoria: EUROBASK. Recuperado de: http://bit.ly/19A7Q69.

García Canclini, Néstor (1999). La globalización imaginada. Buenos Aires: Paidós.

Magallón, Carmen (2012). Contar en el mundo. Una mirada sobre las Relaciones Internacionales desde las vidas de las mujeres. Madrid: Horas y horas.

Mut Montalvá, Elena (2016). El activismo de las refugiadas políticas colombianas. Tesis doctoral inédita. Recuperado de: http://roderic.uv.es/handle/10550/49830

Mut Montalvá, Elena (2016). El activismo transnacional de las refugiadas políticas colombianas en España y su contribución al proceso de construcción de la paz. III Congreso internacional de Estudios de Desarrollo, Zaragoza.

Suárez Navaz, L. (2007). La perspectiva transnacional en los estudios migratorios. Génesis, derroteros y surcos metodológicos. Ponencia en el V Congreso sobre la Inmigración en España. Migraciones y Desarrollo Humano. Valencia. 
VIII Informe sobre violencia sociopolítica contra mujeres, jóvenes y niñas en Colombia 2007-2008. Mesa de Trabajo Mujer y Conflicto Armado. Bogotá: Antropos. 


\title{
Cuadernos de Trabajo Social
}

ISSN: 0214-0314

http://dx.doi.org/10.5209/CUTS.56491

\section{Mejorando la seguridad de los refugiados: una propuesta de intervención del Trabajo Social clínico}

\author{
Ana María Huesca González; ; Silvia Giménez Rodríguez²; María del Pilar Quicios García ${ }^{3}$
}

Recibido: 15/06/2017 / Revisado: 21/07/2017 / Aceptado: 27/12/2017

Resumen. El presente artículo persigue dos objetivos fundamentales: por un lado, tratamos de mostrar la inseguridad en la que viven las personas refugiadas; por el otro, pretendemos señalar la posibilidad de utilizar el Trabajo Social clínico como herramienta para rebajar los efectos que pueda producir tal inseguridad en sus vidas. Para alcanzar el primero de los objetivos, se describe brevemente el panorama internacional que supone la llamada crisis de los refugiados en Europa para exponer, desde la perspectiva de esa realidad, la necesidad de considerar cómo está presente la inseguridad en la vida de estas personas, unas veces como inseguridad objetiva (guerra, violencia, agresiones) y otras en forma de incertidumbre sobre un futuro que se les plantea incierto. Respecto al segundo de los objetivos, se realiza una exposición sobre cómo el Trabajo Social clínico interviene en los procesos de incertidumbre e inseguridad de las personas refugiadas desde una perspectiva psicosocial interdisciplinar en una misma intervención, haciéndose eco de las distintas etapas por las que pasa el proceso migratorio.

Palabras clave: seguridad; incertidumbre; refugiados; Trabajo Social clínico

\section{[en] Improving refugee security: a clinical social work intervention proposal}

\begin{abstract}
This article pursues two fundamental aims: first, to show the insecurity to which refugees are subject, and second, to highlight the possibility of using clinical social work as a tool to reduce the impacts that such insecurity may have on their lives. In order to achieve the first of these aims, we briefly describe the international situation resulting from Europe's refugee crisis to explain the need, from the perspective of this reality, to consider how insecurity is present in the lives of these people. This sometimes manifests as objective insecurity (war, violence, attacks) and on other occasions takes the form of uncertainty over an unpredictable future. With respect to the second aim, we explain how clinical social work offers single interventions from an interdisciplinary psychosocial perspective in processes involving uncertainty and insecurity for refugees, echoing the various phases of the migratory process.
\end{abstract}

Keywords: security; uncertainty; refugees; clinical social work

Sumario: Introducción. 1. Conceptos básicos y aproximación estadística al problema. 1.1. Conceptos básicos en torno al refugiado en Europa. 1.2. Los datos sobre refugiados que alarmaron a Europa. 2. La "crisis de los refugiados" y la seguridad. 2.1. La seguridad desde la perspectiva teórica de la seguridad humana. 2.2. La seguridad de los refugiados en el centro de la reflexión. 3. La intervención con personas refugiadas y el Trabajo Social clínico. 3.1. Delimitación y enfoques teóricos desde el trabajo social clínico con personas refugiadas. 3.2. Intervención psicosocial desde el trabajo social clínico con personas refugiadas. 4. Conclusiones. 5. Referencias bibliográficas.

Cómo citar: Huesca González, A. M.; Giménez Rodríguez, S. Quicios Garcia, M. P. (2018) Mejorando la seguridad de los refugiados: una propuesta de intervención del Trabajo Social clínico, en Cuad. trab. soc. 31(1), 69-83.

\footnotetext{
Universidad Pontificia Comillas de Madrid, España ahuesca@chs.upcomillas.es

2 Universidad Rey Juan Carlos, España silvia.gimenez@urjc.es

3 Universidad Nacional de Educación a Distancia, España mariapilarquicios@gmail.com
} 